\title{
FETID SPIRILLAR BRONCHITIS AND PULMONARY GANGRENE
}

\author{
P. NOLF, M.D. \\ LIÉGE, BELGIUM
}

Castellani ${ }^{1}$ noted in soldiers of the Italian Army the not infrequent occurrence of an affection of the respiratory tract which he had described previously at Ceylon, and of which he had recognized the specific agent. This affection he had termed bronchospirochetosis. It appears under two aspects: A form of short duration, lasting from one to three weeks, and running its course after the fashion of an acute bronchitis, with fairly high temperature during the early days; a form with a prolonged course simulating a chronic bronchopneumonia of long duration, several years in most cases, but sometimes taking on a malignant character, with irregular fever, hemoptysis, progressive enfeeblement and emaciation, and lethal termination.

The specific parasite is a spirillum, Spirochaeta bronchialis, very polymorphous, from 4 to 30 microns long, 0.2 to 0.6 micron in thickness, presenting from two to eight undulations and sometimes more, the extremities of variable shape, often pointed and without flagella.

$\mathrm{H}$. Violle ${ }^{2}$ published an article on an affection of the respiratory tract characterized by bloody expectoration, which he termed hemorrhagic bronchitis. The secretion of the bronchial mucosa is abundant, nearly a quarter of a liter in twenty-four hours. It is tenacious, stringy, viscous, homogeneous, of a bright rosy color, resembling gooseberry juice. It is sufficient, says Violle, to have seen it once to escape the danger of confounding it with tuberculous hemoptysis. On examination of the chest, the results vary: sometimes nothing, sometimes a slight enfeeblement of the vesicular murmur, sometimes signs of a simple bronchitis with or without areas of pulmonary congestion; sometimes, finally, very clear cut signs of chronic bronchitis and emphysema. The general condition is good, often, indeed, very good; there is no fever; the expectoration contains spirochaetes in great abundance. The organisms show an extraordinary polymorphism, and this variation in their shape and dimensions is one of their special characteristics.

1. Castellani, A.: Note sur la "Broncho-spirochaetose" et les bronchites mycosiques, affections simulant quelquefois la tuberculose pulmonaire, Presse méd. $25: 377,1917$.

2. Violle, H.: La spirochaetose bronchopulmonaire (bronchite sanglante), Rev. gén. de clin. et de thérap. 32:144, 1918. 
This affection, for which the author suggests the name of hemorrhagic bronchitis with spirochetes, justifies, generally a favorable prognosis. After ten or fifteen days the expectoration ceases to be mucohemorrhagic, and complete recovery follows fairly rapidly. Nevertheless, relapses, as in the acute form described by Castellani, are surprisingly frequent, and the disease may take on a chronic course with varying periods of exacerbation.

During the war I described, in collaboration with Dr. P. Speh $1,^{3}$ an acute affection of the respiratory tract, the clinical aspect of which is that of a bronchitis or a bronchopneumonia with the special characteristic that the breath of the patient and his expectoration becomes fetid rather early in the course of the development of the disease; the etiologic agent appears to be a spirillum. This malady, so far as we know, has not been observed elsewhere. It would seem to us to be distinguished from the bronchospirochetosis of Castellani and from the bronchitis due to the spirillum described in France by $\mathrm{H}$. Violle.

In my opinion the disease observed by us is not of tropical origin. It is probable that it must be met with in times of peace among the civil population of countries of temperate climate. It has seemed to me that it might be interesting to bring our observations to the attention of the English speaking medical public.

CASE 1.-Dec. . . . A., aged 25, infantryman, fell ill, April 23, 1917, and experienced suddenly, a sensation of general malaise, with headache, pains in the extremities and loins. Cough soon followed, with frothy, whitish expectoration, and a few days later laryngitis. On entry into the hospital, April 29, the patient complained of headache and of general pains. Temperature from 38.6 to 39.4 C.; pulse accelerated, from 104 to 112 ; respiration, quiet; cough, paroxysmal, fatiguing and accompanied by mucopurulent expectoration, whitish gray, of a very fetid odor. There was laryngitis; the voice was hoarse; the naso-pharynx was intact. On examination of the thorax the resonance was normal. Auscultation showed a few sibilant râles, especially at the right base. The general condition was satisfactory.

In the succeeding days the bronchitis increased, the cough became more frequent as well as the expectoration, the fetor of which increased. May 4, the percussion note was diminished at the right base, and auscultation revealed, chiefly at this point, numerous subcrepitant râles. The temperature fell below 37 C., May 9 (seventeenth day) and did not rise again until May 20 (twentyeighth day) when, without apparent cause, there was a slight congestive attack at the right base; at the end of two days the temperature fell again to normal. The local trouble was slow in improving. The râles diminished after May 11 but did not disappear until the middle of June. Laryngitis persisted until the end of the same month.

After May 5, microscopic examination of the sputum revealed for about three weeks the presence of numerous spirilla. Their number together with the fetor of the expectoration diminished after May 20. They disappeared May 25, but the breath of the patient remained fetid until May 29.

3. Nolf, P., and Spehl, P.: La bronchite fétide à spirilles, Arch. méd Belges $\mathbf{7 1}: 1,1918$, 


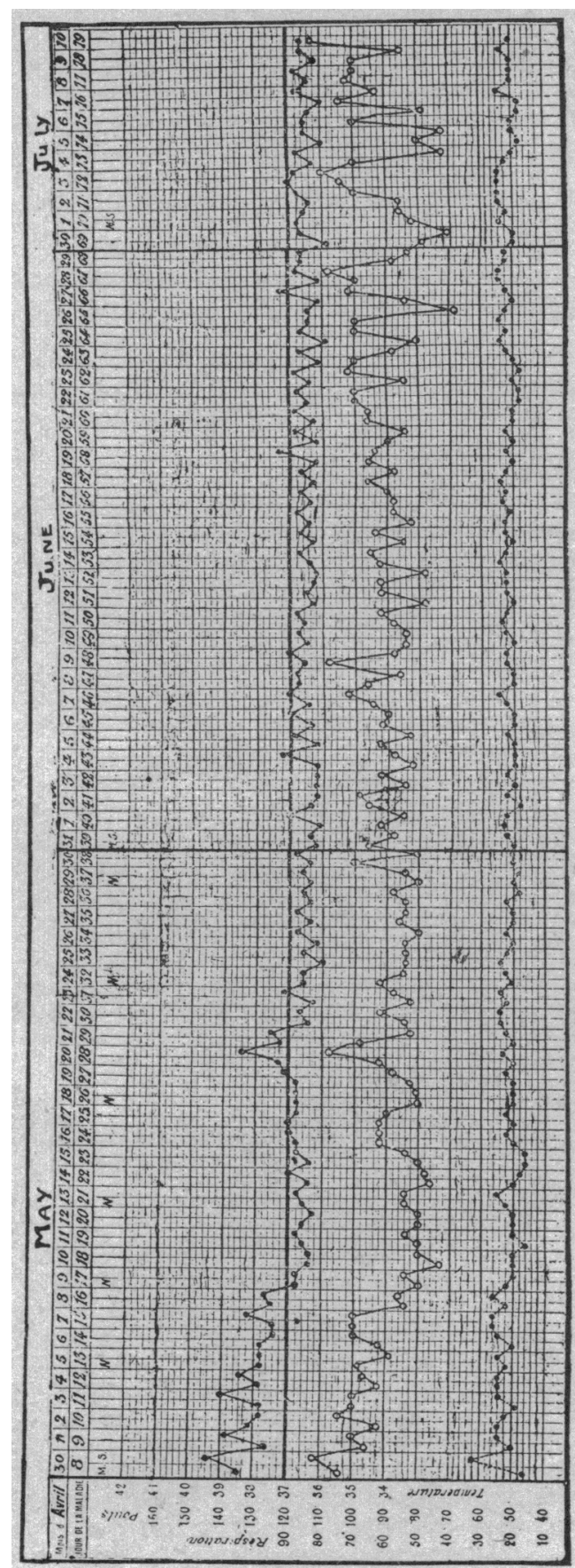


Treatment consisted of warm, moist packs for the chest, with application of tincture of iodin in enemas of creosote and in intravenous injections of novarsan, repeated from $\mathrm{May}^{*} 5$ to the end of the month, every five days, in a dose of $45 \mathrm{cg}$. The amelioration of the general condition became apparent after the first injecton and much more so after the second. The local lesions were not influenced so rapidly. Later, heliotherapy appeared to accelerate convalescence.

For the laryngitis insufflations of sodium benzoate were relatively inefficacious.

CASE 2.-Theun., aged 27, infantryman, suffering from a simple cold, complained on June 29, 1917, of general malaise, thoracic pain, headache and a little difficulty in swallowing. He coughed, expectorated moderately and showed a little fever.

Entering the hospital July 6 , on the first clinical examination there was noted a rather elevated temperature (from 38 to $39.5 \mathrm{C}$.), a pulse of 100 and slight increase in the size of the spleen which reached the costal border on deep inspiration.

The arterial pressure was normal (155/80, Pachon). Examination of the thorax was negative. Chemical examination of the urine, including Diazo reaction and cultures, blood culture, Widal reaction, search for malarial parasites, were negative. There was a slight leukopenia (from 4,400 to 4,800 leukocytes); the blood formula (lymphocytes 19, mononuclears, medium 5, large 1 , intermediate 1 , polymorphonuclear neutrophils 13 , eosinophils 1 ) was. normal.

Up to July 17 (nineteenth day) the fever oscillated continually between 38 and $39.5 \mathrm{C}$. The patient raised, without particular auscultatory signs, a moderate fetid bronchial expectoration which did not contain tubercle bacilli but spirilla in considerable numbers. From this time on a series of injections of novarsan (from 30 to $60 \mathrm{cg}$.) was begun, at intervals of four days, and as adjuvant medication, there was prescribed a daily enema of creosote. After six injections, representing only about $1.95 \mathrm{gm}$. of novarsan, the spirilla disappeared from the expectoration:

However, this treatment remained without action on the general condition of the patient, which became aggravated, or on the temperature, which continued to oscillate between 38.5 and $39.8 \mathrm{C}$.

August 20 (fifty-second day) a laryngitis appeared. The fetid expectoration increased, medium moist rầles appeared at both bases. The pulse increased to 125 , the respiration to 30 . Intravenous injections of peptone, digitalin and strychnin were added to the previous medication. Despite all, the condition of the patient became worse, and death followed September 9 (seventy-third day).

At the necropsy there was edema of both lungs, but no tubercles. At the hilum of the right lung were four enlarged glands. In the pulmonary tissue were some miliary abscesses which do not contain spirilla, tubercle bacilli or giant cells. The spleen was enlarged. The heart, liver, kidneys, intestines were normal.

CASE 3.-Dav. J., aged 26, detached for service at a hospital, fell ill suddenly August 20 . On this date he showed a general malaise, depression, chills, pain in the chest, dyspnea, cough and expectoration.

On entrance (August 23) the patient was much prostrated; the body was covered with cold sweat; the face and extremities were cyanotic The temperature was $39.5 \mathrm{C}$; the pulse and respiration were accelerated; the cough was frequent, expectoration was scanty, mucopurulent, colorless, odorless and aerated. The tongue was moist and white; there was complete anorexia. The urine was deeply colored and showed traces of glucose and albumin.

The upper respiratory tract was normal; percussion of the lungs showed nothing suspicious. On auscultation, disseminated sibilant râles could be heard. Hot packs were applied to the chest and this was painted with tincture of iodin. During the following days, the fever remained elevated; pros- 


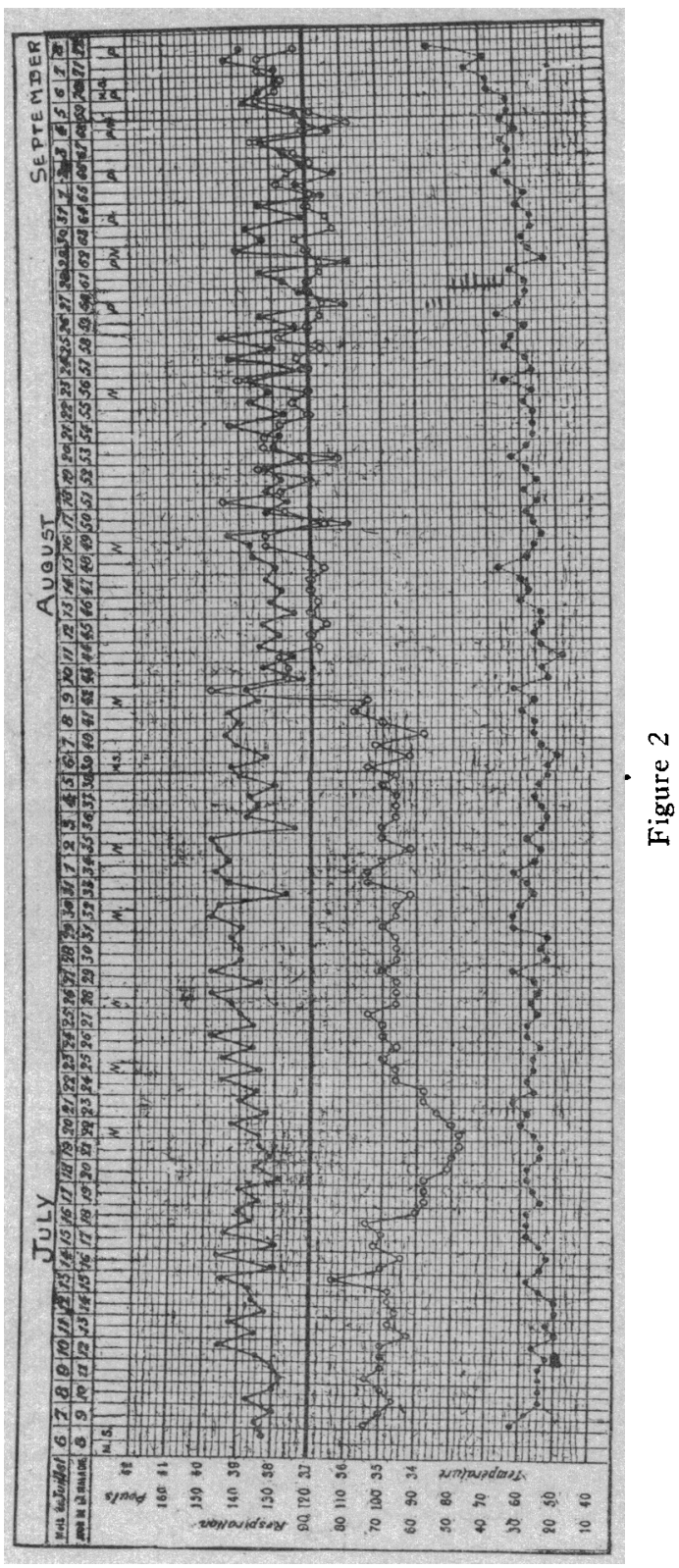

Downloaded From: http://archinte.jamanetwork.com/ by a Monash University Library User on 06/16/2015 
tration and dyspnea persisted; the sibilant râles diminished greatly, but the expectoration increased, became greenish in color and of a foul odor. Microscopic examination made August 26 revealed spirilla in moderate numbers, but no tubercle bacilli. A series of intravenous injections of novarsan was begun (60 cg. on August 26 and 29 and on September 5 and 12) and peptone was given intravenously as an adjuvant $(1 \mathrm{gm}$. on August 31 and September $.2,4,6,8,10,12$ and 17 ).

The spirilla disappeared after September 3, but the expectoration remained fetid up to the middle of the month. Gradually, the pulmonary symptoms receded, the general condition improved, and recovery was complete at the beginning of October.

CASE 4.-Picr . . M., aged 24, infantryman, was taken ill Jan. 13, 1918, with general fatigue, left pleurodynia, severe chill, fever and abundant -sweating. This was followed by cough and rusty expectoration. He entered the hospital, January 16, with symptoms of congestion at the base of the left lung. The temperature was $39.5 \mathrm{C}$; the pulse and respiration were accelerated. Moist warm packs for the chest were prescribed.

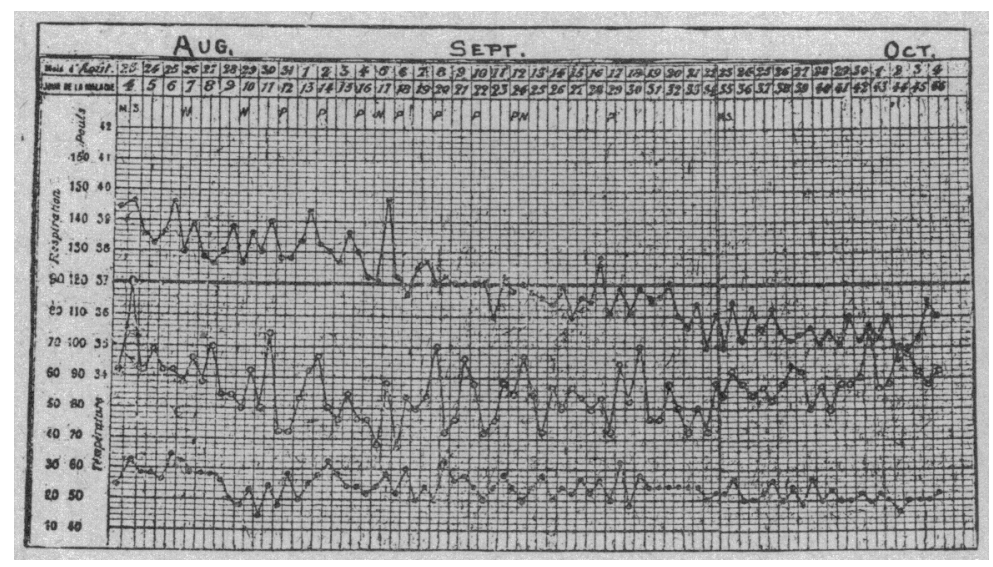

Figure 3

Two days afterward, moist râles were heard at both bases; in the right scapular region there were crepitant râles and slight dulness.

The breath became fetid. The expectoration was mucopurulent and of a greenish color. A daily enema of creosote, $2 \mathrm{gm}$. to 100 c.c., and an intravenous injection of $30 \mathrm{cg}$. of novarsan were prescribed. Examination of the blood revealed 15,500 leukocytes per c.mm., and a leukocyte formula of 9 lymphocytes, 5 medium sized mononuclears, 5 large, 2 intermediate forms, and 79 polymorphonuclear neutrophils. The sputum showed occasional spirilla in addition to an abundant and varied bacterial flora. Injections of novarsan (45 to $60 \mathrm{cg}$., repeated every five days) and intravenous injections of peptone were continued to . March 18 . The temperature fell irregularly to February 6, rising several days afterward and remaining between 37 and $38 \mathrm{C}$. The auscultatory signs (moist râles, fugitive areas of blowing breathing corresponding to areas of bronchopneumonia, with areas of dulness or slight loss of resonance) continued up to February 7. After this date there remained only signs of bronchitis. The general condition improved progressively, keeping pace with the condition in the chest. A blood examination made February 11 showed 85 per cent. hemoglobin (Gowers), 5,460,000 red blood cells and 7,800 leukocytes. Under the influence of pulmonary radiotherapy and heliotherapy, the patient seemed to be entering on convalescence. 


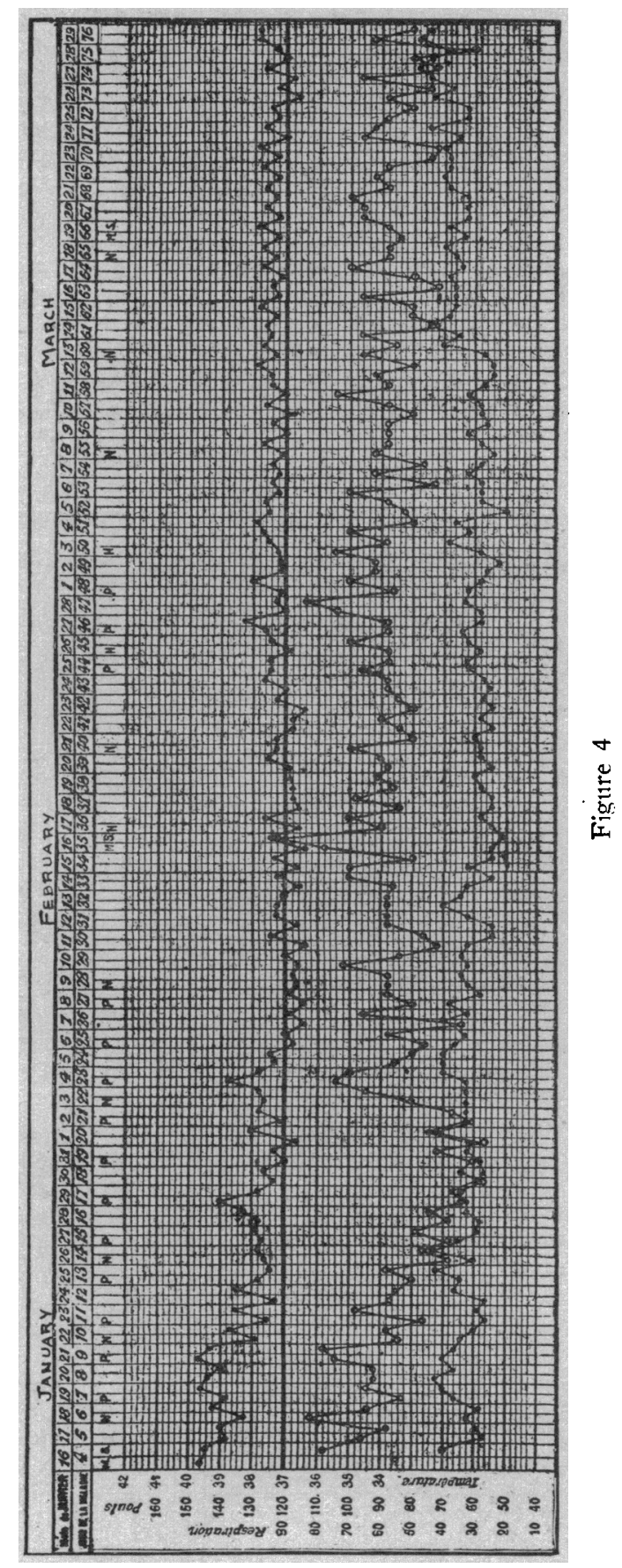

Downloaded From: http://archinte.jamanetwork.com/ by a Monash University Library User on 06/16/2015 
The expectoration remained fetid to the middle of February (sixth injection of novarsan), the spirilla increased to the same time, and then diminished, disappearing with the fetor of the breath about March 8 (tenth injection of novarsan).

CASE 5. - Deh . . . F., aged 27 years, infantryman, subject to winter bronchitis, was seized suddenly Feb. 5, 1918, with several chills, some associated with actual chattering of the teeth. There was headache, nose bleed, nausea and vomiting on five occasions. February 6 and 7 he had colic and three or four liquid stools; slight cough; no expectoration.

The patient entered the hospital February 13. Temperature, 38 to $39.5 \mathrm{C}$.; pulse, 100; respiration, 30 ; nothing abnormal on physical examination. February 14 blood cultures were sterile, no agglutination with dysentery bacillus, typhoid or paratyphoid, but the spleen was palpable. February 15 cultures of the urine and Diazo reaction were negative. On that day, however, the patient began to raise reddish and then rusty expectoration, and examination of the chest revealed dulness at the right base with rough respiration. A few moist râles were heard in this area February 17 . The expectoration remained

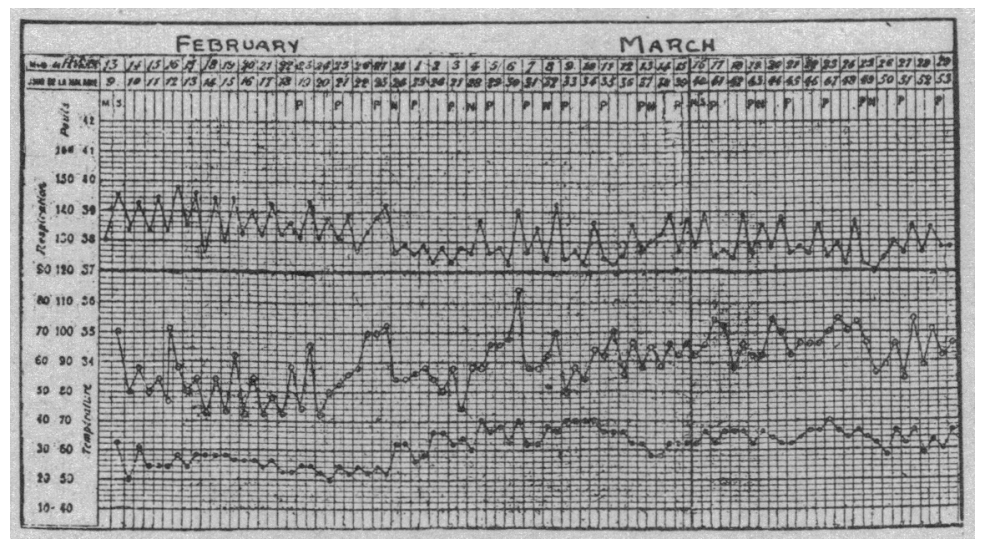

Figure 5

moderate, inodorous, at times mucopurulent, but always contained more or less blood. The temperature remained elevated. New agglutinative tests were made, likewise fresh cultures from the blood and urine; these threw no light on the diagnosis. Because of the intoxicated and prostrated condition of the patient, repeated intravenous injections of peptone were carried out every other day.

February 27, while the condition was still unimproved, the patient began to expectorate a brownish sputum with a pronounced odor of lactic acid. Microscopically, the presence of numerous spirilla (Figs. 7 and 8) was revealed. February 28, along with peptone and wet packs for the chest, a series of intravenous injections of novarsan $(30,45$, then $60 \mathrm{cg}$.) was carried out every five days. In addition, injections of creosote were given, as well as heliotherapy and cod liver oil. During March the pulmonary symptoms continued to remain very slight, the expectoration remained red or brown and still contained numerous spirilla March 16. After March 23 (fifth injection of novarsan) it became paler, more like an ordinary mucous expectoration and contained fewer pathogenic bacteria. The general condition improved slightly. The temperature did not become definitely normal until after March 12, that is until after nine injections of neoarsphenamin. The patient received a tenth injection March 18 and left the hospital entirely convalescent June 21, 1918. Frequent search for the tubercle bacilli in the expectoration was always negative. 
CASE 6.-Van P. H., aged 27, artilleryman, was seized March 12, 1918, with a stitch in the left side and dyspnea. There was fever, cough, abundant expectoration and epistaxis. March 16 the expectoration became fetid. March 20 the patient entered the hospital. Examination of the chest at this time showed slight dulness at the right base, sonorous and sibilant râles disseminated throughout the two lungs, and medium moist râles in the outer part of the left chest. Physical examination was otherwise negative. The temperature ranged from 37.5 to $39 \mathrm{C}$. The pulse was accelerated (96) ; respiration was a little rapid (24). The sputum, examined March 21, was rather purulent, greenish, moderately fetid and contained neither tubercle bacilli nor spirilla. Wet packs were applied to the chest and creosote was administered daily by enema.

March 22 a fresh examination of sputum showed some spirilla. Arsenical treatment was begun. Thereafter the spirilla became more abundant and the fetor of the expectoration increased sharply. At the end of March the disease was still at its height and the general condition remained serious. March 30

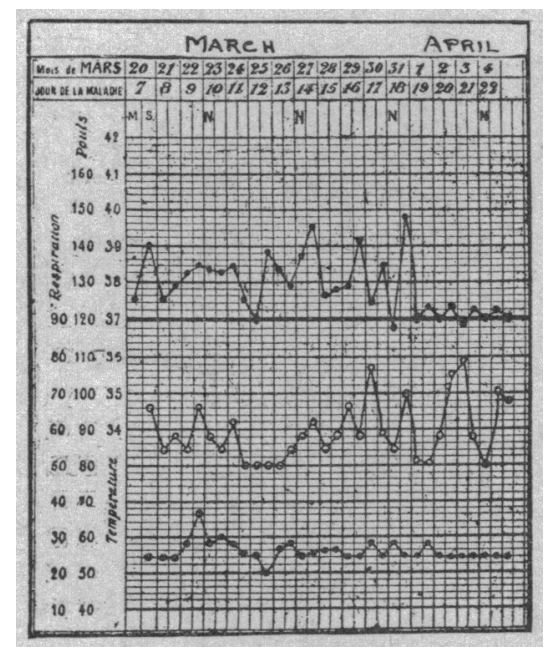

Figure 6

the expectoration contained no more spirilla. March $31,60 \mathrm{cg}$. of novarsenobenzol was given intravenously. After this last injection the general condition of the patient improved; the fever disappeared. For twenty-four hours the patient expectorated more freely. The odor had nearly disappeared from the sputum; there were no spirilla. The soldier left the hospital entirely well April 22, 1918.

In examining the sputum collected in sterile petri dishes after careful rinsing of the mouth, we found in the first four cases and in the sixth, a spirillum, which, although rather variable in its size, presented morphologically an appearance which permitted of its recognition with a little experience. It stained very badly with the basic coloring matters ordinarily used in bacteriology. In order to bring it out, one must have recourse to some mordant. We used the method of Fontana, which gives excellent impregnations when the preparation is spread in 
a sufficiently thin layer, or a procedure described by one of us, ${ }^{4}$ which consists of the use of formol and chromic acid as mordants, staining by formalized violet and reinforcing by Lugol's solution. After this treatment the spirilla are of a brownish-black color (Fontana) or of a bluish-black (Spehl) on the colorless background of the preparation. Their dimensions are variable. Their length varies from 8 to 12 microns, their thickness is about that of Vincent's spirillum; it is a little greater in the preparations stained by Fontana's method. The extremities are tapering and appear not to carry flagella, at least we have never seen them in our preparations. The convolutions are short, sometimes regular, generally irregular, some being much less well marked than their neighbors, which results, perhaps, from the circum-

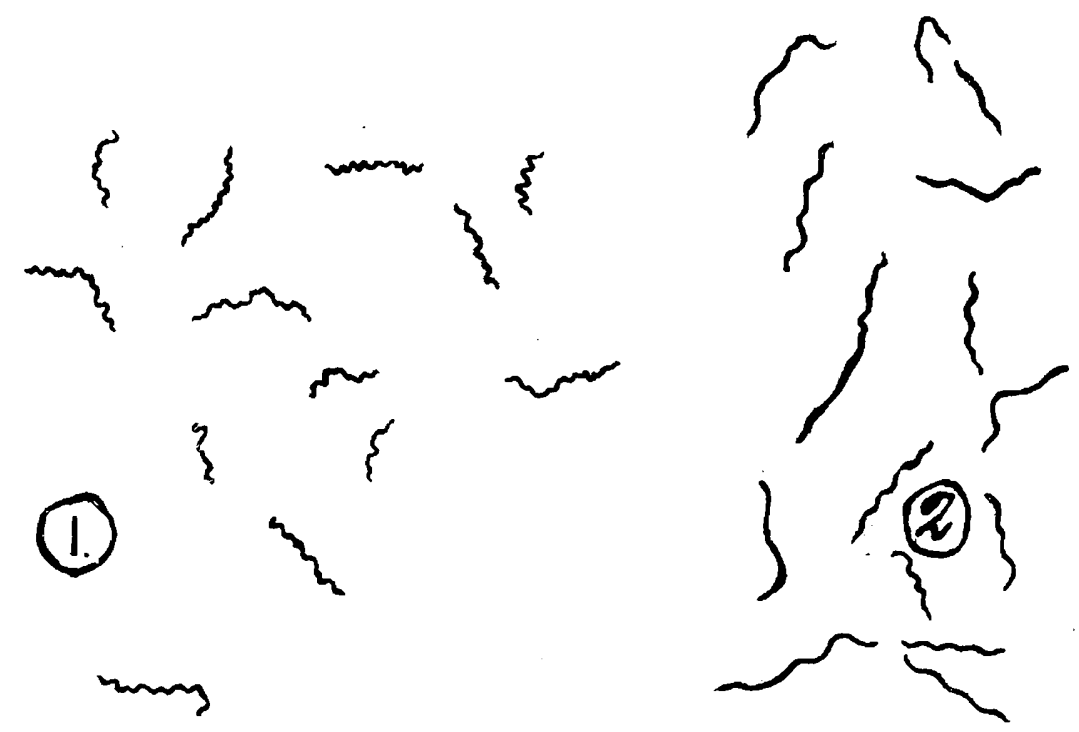

Fig. 7.-1. Spirilla of fetid bronchitis, found in the expectoration of all our patients. (Drawing made with camera lucida, $\times 750$.) 2. Spirilla of Vincent. (Drawing made with camera lucida, $\times 750$.)

stance that the parasite generally takes on a sinuous form, each one of sinuosities including a greater or less number of convolutions. The usual number of convolutions is from seven to eight for the adult forms. Exceptionally, we have counted as many as ten and eleven. What seems to us especially characteristic from the point of view of form is the coincidence of elbows or undulations of large size and of short, crowded convolutions, which gives to the spirillum a particularly tortuous character (Fig. 7). The accompanying figure drawn with the camera lucida, reproduced fairly faithfully the appearance of the preparation.

4. Spehl, P.: Procédé de coloration des spirilles par le violet-formolé, Compt. rend. Soc. de biol. 81:305, 1918. 
In examining these two figures, one may see morphologic differences which distinguish clearly the spirillum of fetid bronchitis from the spirillum of Vincent.

So far as we know few spirochetes take this aspect. With some experience it is easy to differentiate the micro-organism which we have found in our cases of bronchitis from the two known spirilla of the buccal cavity, such as the spirillum of Vincent (Spirillum refringens) and Spirochaeta dentium). We do not, then, wholly share the opinion of Castellani and Violle, each of whom insists on the extreme polymorphism of the spirochetes which they have described. Indeed, we have succeeded in finding, alongside of these forms, which we regard as characteristic, spirillum of aberrant form, sometimes in great numbers.

Our first observation was made prior to these two publications. It dates back to April, 1917: Between April, 1917, and July, 1918, we observed ten other cases among about 5,000 patients who passed through our first line hospital during this period. In our eleven patients there was also bronchitis, or more frequently bronchopneumonia, as in those of Castellani and Violle, but the clinical appearance and evolution differed at several points from the affection described by those authors.

In nine of our eleven cases the disease was primary. It seized the men in good health, and generally had a sudden onset, characterized by one or more chilly sensations or shaking chills. The patient suffers from general malaise, headache, pains in the extremities and loins. The spleen was palpable in two instances. The cough generally produced pain in the chest without an actual stitch. In one case there was epistaxis, scanty but repeated. In several cases the pulmonary symptoms were very slight. in the earlier days of the disease, which appeared rather as a general affection than as a malady of the respiratory tract. Even after the symptoms related to the respiratory tract have developed further and taken a prominent place in the picture, there may, at the outset, be nothing characteristic, the local signs being those of an ordinary bronchitis and the expectoration mucopurulent, frothy, without special morphologic or bacteriologic characteristics. But there is one characteristic common to all the cases; namely, that the disease never shows the least spontaneous tendency to recovery.

The fever, generally irregular, is at the outset elevated, or tends progressively to rise, the general condition becomes aggravated, the patient loses strength, is feeble, out of breath on the least effort, while the face and extremities assume a more or less marked cyanosis. In the chest the signs of bronchitis, indefinite at first, become clearer and more extensive; in the posterior parts of the lungs zones of dulness or diminished resonance develop, localized commonly at the bases; the 
expectoration becomes more and more abundant; it is slightly aerated, purulent rather than mucopurulent, of a greenish yellow color, and an important sign - it becomes fetid while the breath takes on a repulsive odor. This fetor of the breath and expectoration has been manifested in all of our patients. In the grave cases it existed at the outset and had a cadaveric character. With the spasms of cough it spread to some distance, and distressed the patient's companions in the ward at a distance of several meters. Sometimes the air of the whole room was poisoned.

In the milder cases the fetor was not evident in the early days of the disease, or was too slight to attract attention. It was only after several days that it was perceived by the hospital personnel, or sometimes by the patient himself. But, as has been said, it always appeared at some period in the evolution of the malady, generally precociously. This characteristic is all the more notable.in that it is not pointed out by Castellani or Violle.

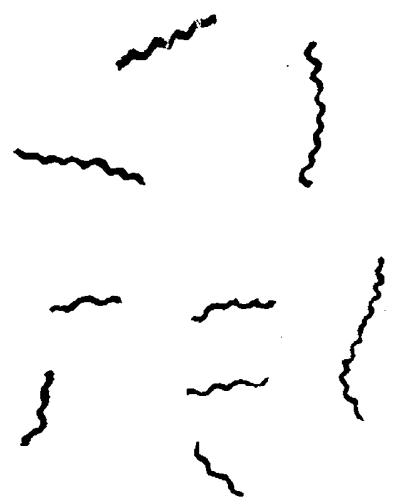

Fig. 8. Spirilla in sputum in Case 5. (Camera lucida drawing, $\times 750$.)

The expectoration of our patients is distinguished further from the bronchial secretions of patients observed by Violle. It was hemorrhagic in only one case. In this case it had the characters of glary, viscous masses with a homogeneous rose violet color. Later, it took on a brownish hue. In another patient, the expectoration was rusty in the first days of the disease, but became rapidly mucopurulent. Apart from these two cases the bronchial secretion of our patients always had the color and consistency described in the brief résume of the history of six persons attacked by the primary form of the disease. Thus, in Case 5, which is, by the way, the only instance of expectoration regularly bloody in the early days of the disease, we found, for the greater part, forms a little different from those which have been described, generally shorter, less tortuous, a little thicker, and a little less tapering at the extremities (Fig. 8). 
Alongside of these there were, however, fewer spirilla having the characteristics of those which we found in all our other patients. It is difficult to express a definite opinion as to the question whether the micro-organism represented in Figure 8 is different from that shown in Figure 7 (1).

In one of the patients, where the affection was secondary (Case 7) and in whose expectoration the spirilla were particularly abundant, there were, alongside of the ordinary forms, a certain number of spirilla which, from their appearance, recall the spirillum of Vincent. In this case, which was one of particular gravity, the expectoration was extremely abundant and of a repulsive fetor. Possibly, the bronchial lumina had been invaded secondarily by the micro-organisms of the mouth, which in this very sick man, may have found in the bronchial secretions an excellent culture medium.

An interesting point is the greater or less abundance of spirilla in the expectoration. In certain patients they are very abundant. They may be found, so to speak, in every field of the microscope, sometimes several in each field, even in very thinly spread preparations. But it is not always thus. In other instances the micro-organisms are much rarer. One must sometimes examine a large number of fields before finding one. In a general way, one may say that the gravity of the affection is in direct proportion to the number of the spirilla. In the fatal cases they are as abundant in the bronchial mucus as is the spirillum of Vincent in the detritus which covers the ulceration of the disease tonsil.

It has seemed to us that the more numerous they were, the more fetid was the breath and the expectoration, and it was because in our first patient this fetor was very striking and the number of spirilla great, that we thought immediately of seeking a relation between the one and the other, and recognized the possibility of the pathogenic rôle of the spirillum. On the other hand, when, under the influence of treatment, the disease tends toward recovery, improvement goes hand in hand with the diminution of the spirilla in the secretion, a diminution which becomes evident even when the general characters of the expectoration, excepting the odor, have as yet shown no change.

In summary we found in our six patients with a primary fetid bronchitis a spirillum which seems to us to be the same in all cases, except for the circumstance that in Case 5 it was associated with a second form.

From a clinical point of view, the affection is sufficiently characteristic to permit its recognition after several days of observation at least. That which distinguishes it, as has been said above, is the high remittent fever, which has no tendency to fall spontaneously, despite a severe regime (rest in bed, liquid food, wet packs to the thorax, expec- 
torants or balsamics) progressive loss of strength, dyspnea on the slightest effort, more or less well marked cyanosis and the peculiar character of the breath and expectoration. All of our last cases have been recognized clinically before confirmation from the laboratory. As. a matter of fact, we hold that the only possible confirmation is the habitual presence in the sputum of a spirillum with the staining characteristics and the form described.

When one examines carefully the bronchial expectoration of patients affected with acute or chronic inflammation of the bronchi or of the pulmonary tissue, one may discover occasional spirilla, but this is rather an accidental event. The micro-organisms are rare, and have not, from the standpoint of their shape, the characters that we have found constantly in all of our cases. The question which arises is as to what is the etiology of this affection and how it spreads. Is it a malady analogous to whooping cough or measles, whose parasite exists only in those who are affected with or in the period of incubation of the disease, or must it be placed alongside of pneumonia whose microorganism is to be found in the upper respiratory tract of healthy subjects. Our two cases of secondary fetid bronchitis speak in favor of the second alternative. The first case was that of a man, who, having a gastric ulcer, was obliged to undergo a gastro-enterostomy because of persistent hemorrhage at a time when his resistance was low. Immediately after the operation, there was fever, attributable to a pneumonia at both bases, which from the outset was characterized by a fetid breath and mucopurulent expectoration rich in spirilla. This man had been in the hospital for four weeks, in a ward where no similar case had been treated previously. The affection pursued its course to a fatal issue in twelve days.

The second case was that of a soldier who had been gravely intoxicated by dichlorethylsulphid. The bronchopneumonia produced by the poison took on an infectious character on the fourth day. Little by little, the expectoration became fetid. This phenomenon led to a microscopic examination of the sputum which revealed the presence of very numerous spirilla, but, curiously enough, the spirilla particularly abundant in these two extremely grave and fatal cases, after a short course, were the same in their staining affinities, their dimensions and their form, as those found in the cases of primary bronchitis. We are, therefore, led to conclude that the upper respiratory tract of these two men, normal up to that time, harbored the specific spirillum, and as there is little reason to believe that they were in a period of incubation, since the first had been cared for at the hospital for more than four weeks, we are rather led to conclude that the spirillum of fetid bronchitis may exist as a saprophyte in wholly healthy individuals. In like manner, we may add, that although our patients suffering from the 
primary affection were never isolated, and although they lived for considerable periods in the common wards, we have never observed an instance of contagion. The affection is, however, relatively rare, since we have had only eight cases, ${ }^{5}$ as against eighty-three of pneumonia and bronchopneumonia treated during the same period.

The history follows of two cases of secondary bronchitis:

CASF 7.J. O. R. . . . E., aged 29, in the auxiliary service, for a year has suffered from intermittent gastric disturbance. September 8,1917 , he was seized by vomiting, which continued during the following days and was preceded by pyrosis. Hematemesis occurred on the evening of September 20 and necessitated his entering the hospital on the twenty-first.

On examination, a diagnosis of gastric ulcer was made, and the patient was treated by suitable diet, calcium chlorid and several intramuscular injections of peptone. His general condition being sufficiently improved, he underwent an operation for gastro-enterostomy (ether narcosis) October 25. With the exception of several attacks of vomiting the results of the operation were good.

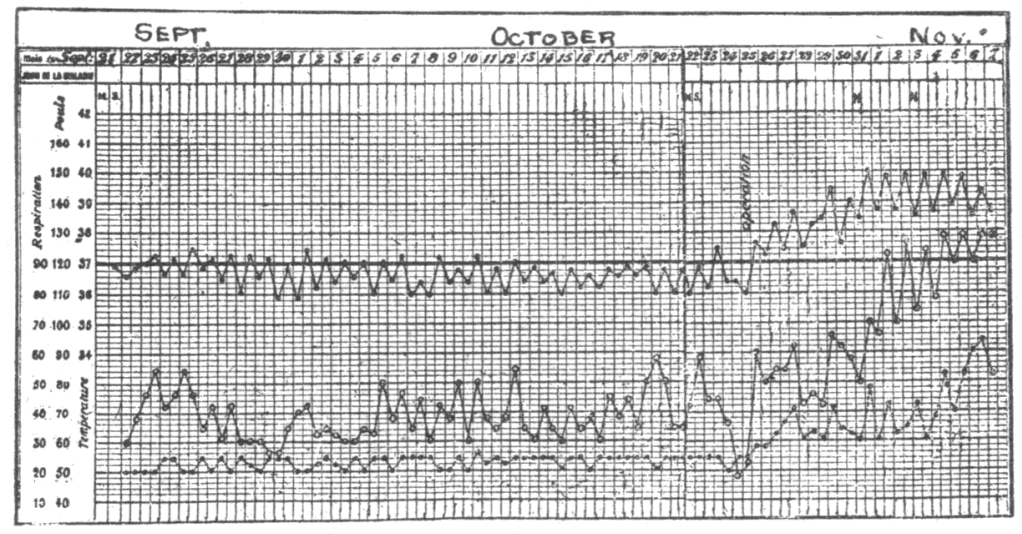

Figure 9

October 27 the patient began to expectorate a little mucus. The cough brought on vomiting. October 28 and 29 the vomiting ceased, but expectoration became mucopurulent and increased in quantity. October 30 the expectoration began to give forth a slightly disagreeable odor, but became clearly fetid on the following day, and was of a frankly purulent character. A microscopic examination made October 31 revealed numerous spirilla. The patient was immediately transferred to a medical ward and received an intravenous injection of $45 \mathrm{cg}$. of novarsenobenzol. Examination of the thorax revealed dulness at the bases of the lungs, especially the left. In places there was pectoriloquy and blowing breathing. Dyspnea was marked and prostration striking. Warm moist packs were applied to the chest. November 1 the fetor increased and this increase continued until his death. The pulmonary symptoms persisted. Digitalin was administered. November 3 a few fine râles appeared in the middle of the left lung in addition to the râles of bronchitis, and another dose of novarsan was administered. November 4 and 5 , the general condition improved and intravenous injections of peptone were given.

5. In April and May, 1918, we had occasion to care for three new cases, and to bring the patients to recovery by intravenous injections of novarsan. 
November 6 , the symptoms became aggravated. The spirilla were always very abundant; the fetor of the breath was evident in the ward; dyspnea was intense; the patient was given oxygen through a nasal tube, and camphorated oil, strychnin and tartar emetic. November 7 there was bilious vomiting. The patient died at 5 p. m.

At necropsy the lungs showed a generalized edema, patchy bronchopneumonia and miliary abscesses, in the pus of which the pathogenic spirilla were found.

CASE 8.-Baud . . . R., aged 25, infantryman, intoxicated by dichlorethylsulphid during the night of November 12 to 13 , entered the hospital on the thirteenth suffering from burns of the face, eyes, scrotum and a general bronchitis. There was a little cyanosis of the extremities, but respiration was quiet and the pulse was not accelerated. Despite hot, wet packs to the thorax, the application of tincture of iodin and Dover's powder, the patient's condition became worse, the fever and pulse increased.

November 15 the respiration was blowing at both bases and accompanied by moist râles and a diminished resonance. Small doses of digitalin and ipecac

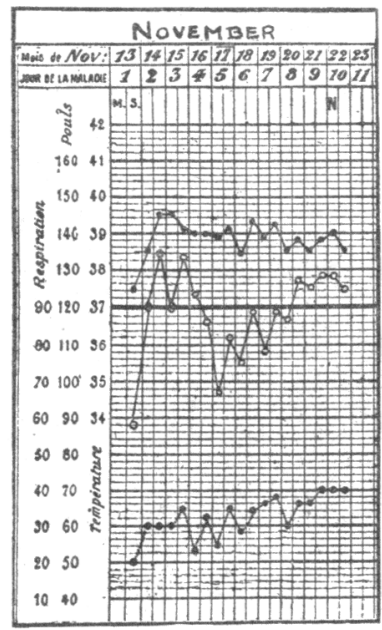

Figure 10

were given. The local signs, the exhaustion and cyanosis persisted, the expectoration became more abundant and took on a purulent character (November 20). Iodotannic syrup and creosote enemas were added to the treatment. November 21 , the patient became definitely dyspneic. He was given oxygen continuously, strychnin and camphorated oil. November 22 the expectoration became fetid. Immediately, the patient received a potion of tartar emetic and an intravenous injection of $60 \mathrm{cg}$. of novarsenobenzol. Microscopic examination of the sputum showed many spirilla. November 23 , his condition was desperate; there was pronounced fetor and numerous moist râles were audible in the chest. Death ensued in the morning.

At necropsy the lungs were generally edematous and congested and there were areas of disseminated bronchopneumonia. The glands of the hilum were enlarged.

If there were still doubt concerning the pathogenic rôle of the spirillum, it would be removed by the curative influence of the organic preparations of an arsenic base. This action is indeed not always 
shown at the outset. Treatment must be long to control the disease definitely. If a series of intravenous injections of novarsenobenzol is made, in doses of from 45 to $60 \mathrm{cg}$., five or six days apart, a change in the clinical picture, rather slow in its development, may usually be made out. The condition, which, up to that time, has grown progressively worse, shows little by little, signs of an improvement, which, interestingly, affect first the elements characteristic of the affection. On the one hand, the fetor of the breath and the expectoration becomes less. On the other hand, there is a diminution in number, or even disappearance of the spirilla under the microscope. During this early period of arsenical treatment, the fever may remain tolerably high, and the local signs in the pulmonary apparatus are little influenced. It may even be that with the continuation of the arsenic treatment, the disappearance of the spirilla is brought about without amelioration of the general or local condition. This occurred in Case 2, which ended fatally. We have observed that the arsenical treatment acts better and more quickly the earlier it is begun.

This circumstance may be explained when one considers that the spirillum is far from being the only micro-organism to be found in the sputum which contains a particularly varied and abundant bacterial flora. The micro-organisms of which this consists probably add their injurious action to that of the spirillum. At the necropsy done in Case 2, both lungs were found dotted with miliary abscesses, of which the pus did not contain spirilla (tubercle bacilli were also absent), although the expectoration of the patient contained many before the beginning of arsenical treatment. It would appear that the lesions produced by the spirilla are severe, and that, invaded by the micro-organisms of secondary infection, they present little tendency to heal. This, perhaps, is the most distinctive character of the affection described in this article. Even after our patients had been rendered apyretic, and after their expectoration from being purulent had become mucous and free from spirilla, they continued to cough, and auscultation revealed signs of an attenuated but persistent bronchitis. We have had to have recourse to the combined action of balsamics, exposure to sun, and respiratory gymnastics definitely to overcome these sequels which always show an extreme obstinacy.

In certain cases we have added to intravenous injections of novarsenobenzol, peptone, administered intravenously every two days. It has seemed to us that this combination was more effective than treatment by arsenic alone (see especially Case 3 ).

We have termed the malady described in this article "fetid spirillar bronchitis," although, in reality, certain forms have from the outset taken on the clinical characters of a bronchopneumonia, and although at necropsy in the fatal cases there have been found either areas of 
bronchopneumonia or pulmonary abscesses. The appellation "bronchitis" seemed to us to be more suitable because, at least in the primary form of the affection, the signs in the respiratory tract were very vague in the early days of the disease, and were those of an acute bronchitis. The examination of the sputum having proved that in addition to a spirillar infection there is added a considerable number of other micro-organisms, it is impossible to settle the question as to whether the extension of the bronchial inflammation to the pulmonary parenchyma is attributable to the spirochete or to the organisms of secondary infection. In the early days of the disease, when it is primary, the fetor may be absent or slight, and the spirilla very scanty in the expectoration. It may even be impossible to make them out at that time. Although the disease is not malignant, it is to be differentiated, however, from an acute bronchitis by a sudden onset with high fever, general phenomena, and especially a complete lack of tendency toward spontaneous recovery. After from several days to several weeks the patient loses his strength, becomes emaciated, his bronchitis merges into bronchopneumonia, the spirilla tend to become more abundant in the expectoration, while the fetor of the breath also becomes aggravated. All the patients observed by us were soldiers in the Belgian Army in the field, who had not lived in tropical countries, and had never been in contact with colonial troops.

For this reason, and for the others mentioned above, we have differentiated the affection from the two maladies analagous and yet distinct one from another, described, one by Castellani, ${ }^{1}$ and the other by Violle, ${ }^{2}$ both of which appear to originate in. warm climates.

If, as we thought, the affection is autochthonous, it remains to be explained why we have had occasion to see a considerable number during the course of a year when, so far as we know, no disease of this sort had previously been described in temperate regions.

The most probable explanation appears to be the following: If the case is benign and remains benign (this eventuality we have not observed, but our observations do not permit us to exclude it), if it ends in recovery spontaneously or favored by treatment, there is considerable likelihood that it may be regarded as an acute catarrh of the upper respiratory tract. If, on the contrary, the malady becomes aggravated and lethal in its course, it will impress the uninformed physician as being an acute bronchopneumonia which, at a given moment, is complicated by pulmonary gangrene.

As a matter fact, the observations at necropsy in our fatal cases do not at first glance plead in favor of this point of view. One observes in the examination of the lungs postmortem, the presence of diffuse congestion and edema, areas of disseminated bronchopneumonia, sometimes multiple miliary abscesses, but no pulmonary gangrene. 
During the summer of 1918 , one of us had occasion to see a patient whose history would seem to bring new evidence toward the solution of the above problem. The following is a résumé of this observation. ${ }^{6}$

CASE 9.-Berch, M., a soldier, aged 32 years, presented nothing abnormal from a hereditary standpoint. He had pneumonia at 17 , acute dysentery one year ago. The history was one of sudden onset June 9, 1918, with general malaise and pain in the back. June 10 he had a stitch in left side in the region of the precordium, with dyspnea. Cough and expectoration appeared on the following day. On entering the hospital, June 13, he had a temperature of $37.2 \mathrm{C}$, pulse 100 , and moderate dyspnea. His general nutrition might have been better. He was thin; his muscles were poorly developed. There was great prostration. The tongue, covered with a thick, whitish coat, tended to be dry. There was pyorrhea alveolaris. The digestive tract was negative. On examination of the chest, there was found diminution in resonance at the left base, and fine, localized crepitation in the left axilla. Throughout the remainder of the chest, dry râles of bronchitis were heard. The heart was negative. His arterial pressure was 16.9 (Pachon). The urine showed a trace of albumin. In the days following the temperature fell gradually, reaching normal June 22, while the signs of bronchopneumonia increased. The left base became dull, bronchial blowing breathing was heard. Later, June 20 , the right base showed similar signs.

Moreover, June 23, fever recurred, the temperature rising to $38.2 \mathrm{C}$., descending gradually toward normal during the following days. June 29, the patient was afebrile, and remained afebrile until July 9, when there was a moderate new febrile paroxysm lasting several days. By July 12 there was again defervescence, of more than one week's duration without marked change in the condition of the bases posteriorly. The patient did not regain his strength. July 22 the fever again recurred, this time to remain. It was very irregular. The local signs did not vary. Expectoration became abundant; it was mucopurulent and fluid. Tubercle bacilli were sought for on various occasions without results. July 25 the patient expectorated sputum mixed with red blood about twenty times. This symptom caused us once more to incline toward the diagnosis of pulmonary tuberculosis, although examination of the chest revealed no lesions in the apices.

The appearance of a new symptom on August 5 definitely settled the diagnosis. On this day the breath and sputum were found to be very fetid. The nurse had made the same observation during the preceding days without thinking of calling our attention to it. The microscopic examination of the sputum revealed spirilla altogether analogous to those described by Spehl in several cases of fetid bronchitis. Nevertheless, despite the repeated administration of novarsenobenzol intravenously, the situation did not improve. August 16 the expectoration became hemorrhagic. August 17, the patient raised pure blood, and died on the eighteenth with abundant hemoptysis.

The necropsy, made by Dr. Colard, showed nothing notable in the heart or digestive tract. The spleen was increased in size and diffluent. No tubercle were found in the spleen, liver or kidneys. The right lung was adherent to the parietal pleura, and the three lobes were glued together by recent adhesions. There was diffuse bronchitis, congestion of the base; no tubercles. The left lung was bound over its whole surface to the parietal pleura by old, thick adhesions. In the upper lobe there was bronchitis without tuberculous lesions. The lower lobe was riddled with numerous cavities filled with foul pus. One of these, situated in the posterior and lower part, was of the size of a large nut. There were, moreover, numerous miliary abscesses. The lung itself was gangrenous almost throughout its lower lobe.

6. Nolf, P.: Un cas de gangrène pulmonaire à spirilles, Bull. de l'Acad. de méd. Par. 80:657, 1918. 
The interesting point about this case lies in the circumstance that it presented at first, the characteristics of secondary spirillar bronchopneumonia, the primary infection being here a grippal bronchopneumonia. There was, however, a clear tendency to pulmonary hemorrhages, and, indeed, it was as a result of an accident of this nature that the patient died. Barring this complication, nothing would have justified a clinical distinction between this case of secondary spirillar bronchopneumonia and the two cases observed above, but the necropsy showed the presence of an extensive gangrene of a lobe of the lung, which was absent, as has been said, in all the cases published by Spehl and me.

It has, therefore, been shown that one may find spirilla similar to those described by us in the expectoration of a patient in which an acute bronchopneumonia becomes complicated at a given moment with pulmonary gangrene. It is probable that this observation will be repeated when in other like cases, spirilla are sought by appropriate methods. It remains to be explained why, in the fatal cases which we have published previously, gangrene was nonexistent.

Two hypotheses may be considered: (1) In rapid diffuse forms, such as in Cases 7 and 8, death may occur before gangrene has had time to develop. (2) In the more circumscribed forms the evolution of which is less rapid, like Case 2, death at a late period seems to have been the result of secondary complications, as is proved by the complete disappearance of spirilla in the expectoration and in the pus of the abscesses-a disappearance brought about probably by arsenical treatment.

If the last hypothesis is sound, the sum of the observations heretofore made would allow us to ascribe to the spirilla a role in the production of gangrene.

In the present state of the question, and so far as our observations justify a conclusion, it would seem, then, that spirillar bronchopneumonia is a malady of temperate climes, and that it has probably been confounded up to the present time, with pulmonary gangrene consecutive to acute or chronic affections of the respiratory tract. Its micro-organism is probably a common inhabitant of the upper respiratory passages, particularly of the mouth and pharynx. In this connection it is interesting to observe that the last mentioned patient suffered from pyorrhea alveolaris at the time of his entrance into the hospital. 